ATLAS Pixel Detector Design For The HL-LHC

\title{
Ben Smart
}

on behalf of the ATLAS Collaboration
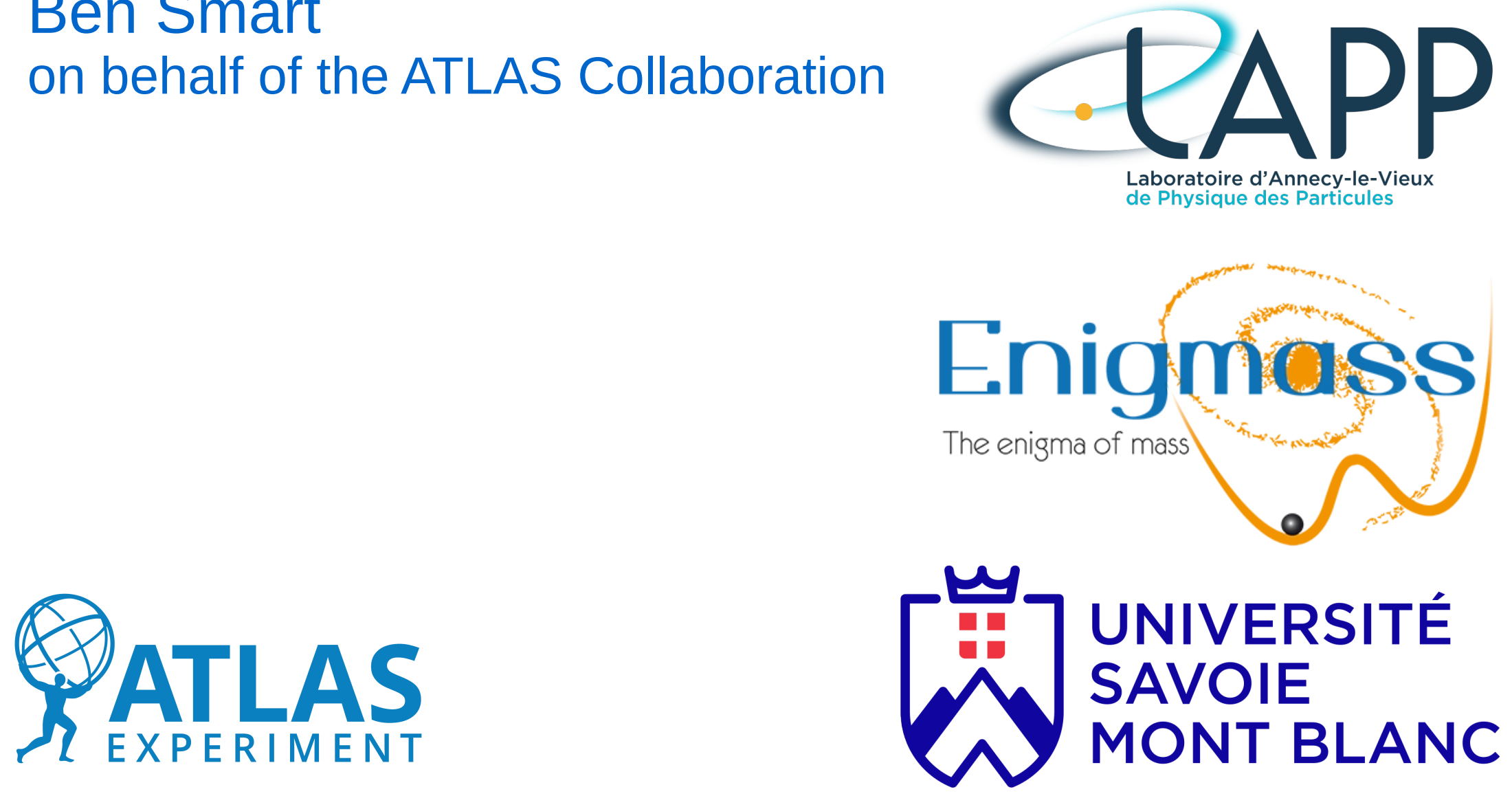
In the future, the LHC will go to higher energy and luminosity

$$
\text { This will be called the 'High-Luminosity LHC' (HL-LHC) }
$$

The ATLAS detector will also be upgraded

\section{The current ATLAS Inner Detector will be removed, and replaced with a new, all-silicon sensor, inner tracker}

This new Inner Tracker will be called the ITk 
- To improve our measurements and searches, the LHC and ATLAS will be upgraded:

\section{LHC / HL-LHC Plan}

High

Luminosity

LHC

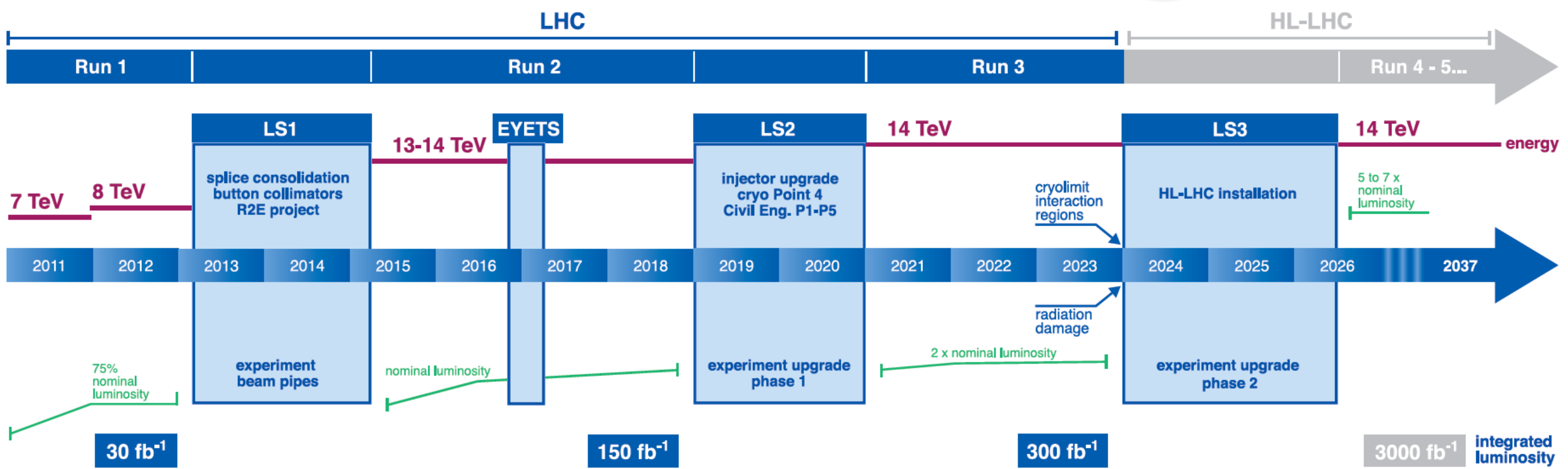

- The LHC will become the High-Luminosity-LHC, to produce $3000 \mathrm{fb}^{-1}$ of integrated luminosity in 2035. Higher Energy $\rightarrow$ benefits searches for new particles.

Higher integrated luminosity $\rightarrow$ benefits precision measurements and studies of rare processes.
- Instantaneous luminosity x5-7

$\rightarrow$ Particle densities $\times 5-7$

- Integrated luminosity x10

$\rightarrow$ Radiation damage $\times 10$ 
- For this talk, I will focus on ATLAS Inner Detector (Silicon pixel) upgrades.

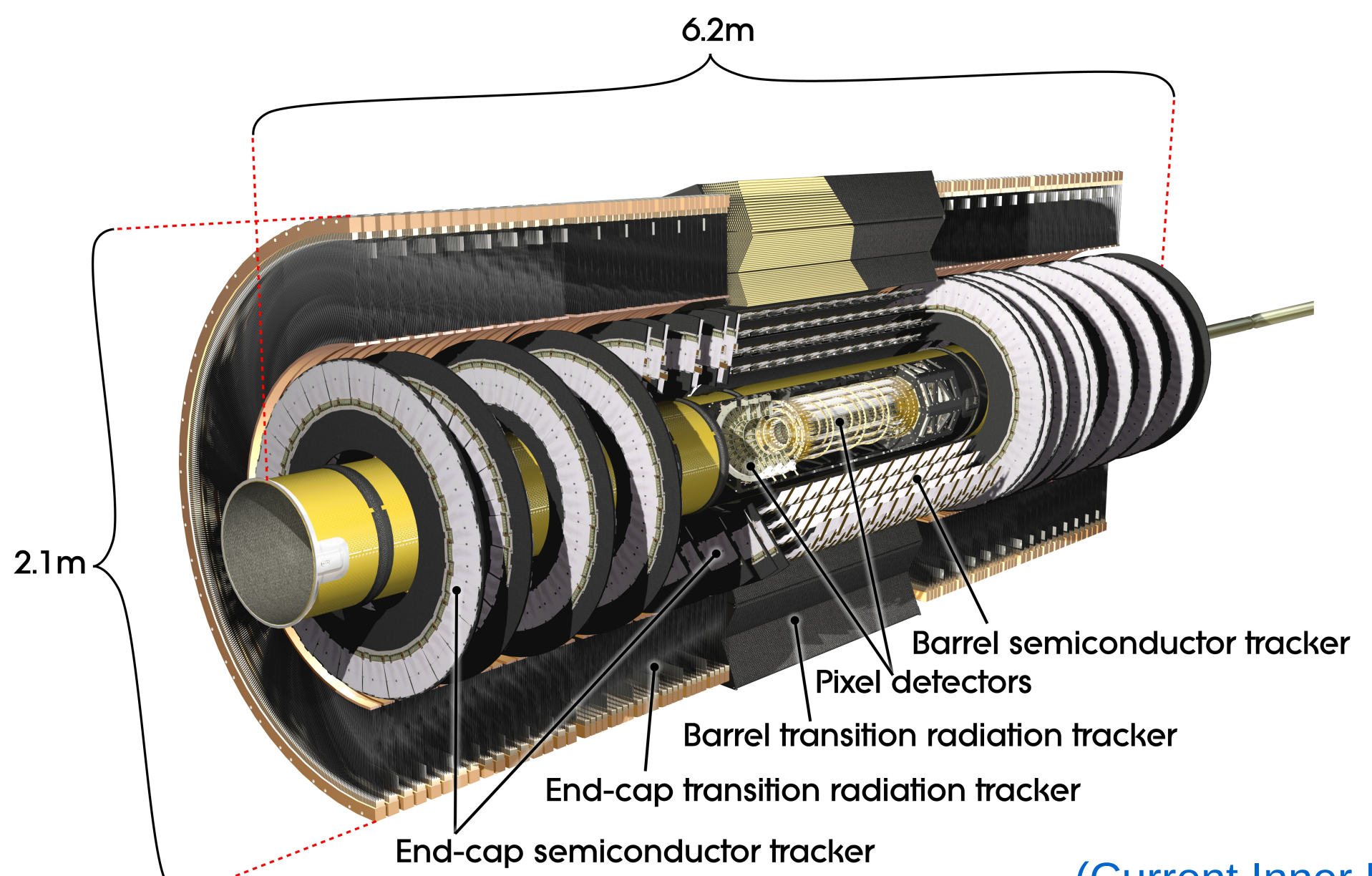

(Current Inner Detector, to be removed) 


\section{ATLAS event, with 25 reconstructed vertices $(\mu=25)$}

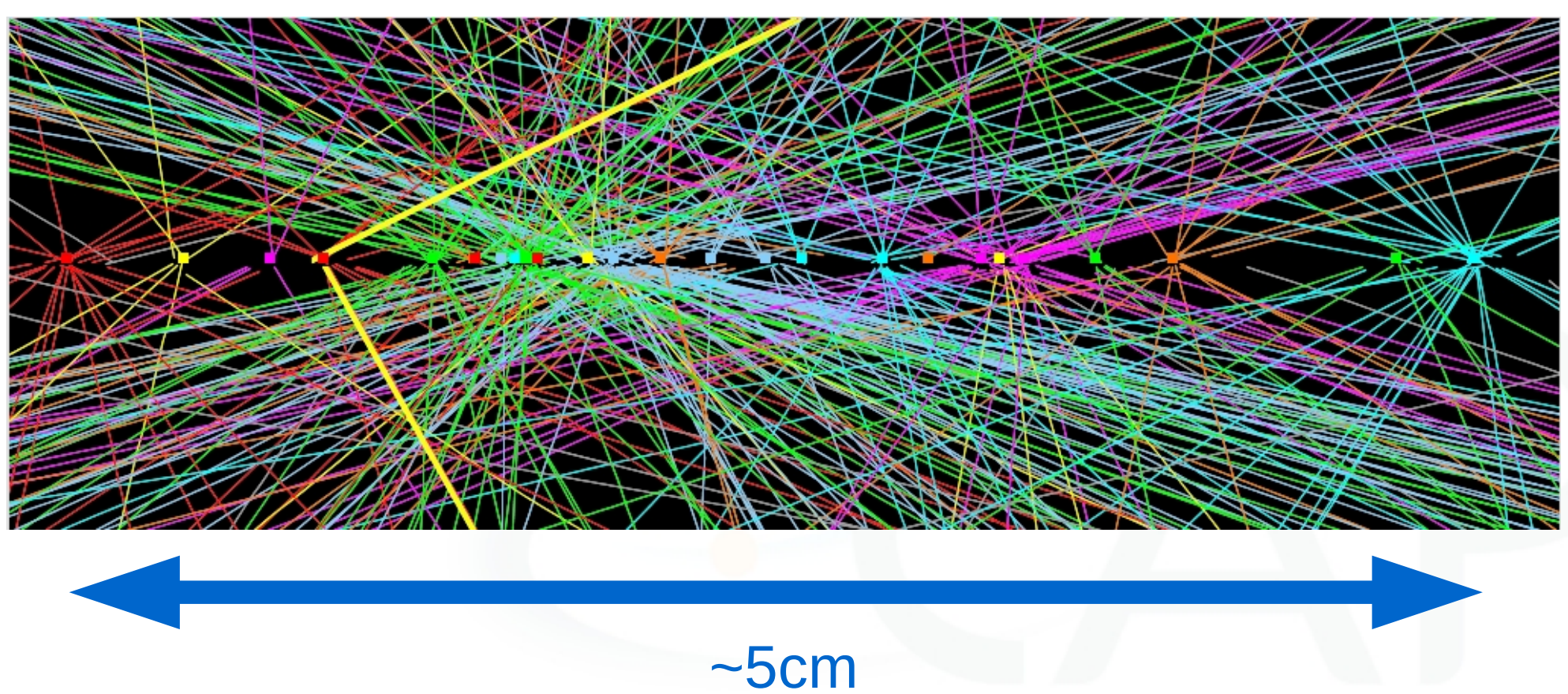

- At the HL-LHC (upgrade phase 2) we expect $\mu=200$ within a similar or shorter space.

- Higher track density requires a better Inner Tracker to maintain tracking and b-tagging performances.

- Existing components will be heavily radiation-damaged by LS3. 
Simulated HL-LHC event, with 200 reconstructed vertices $(\mu=200)$

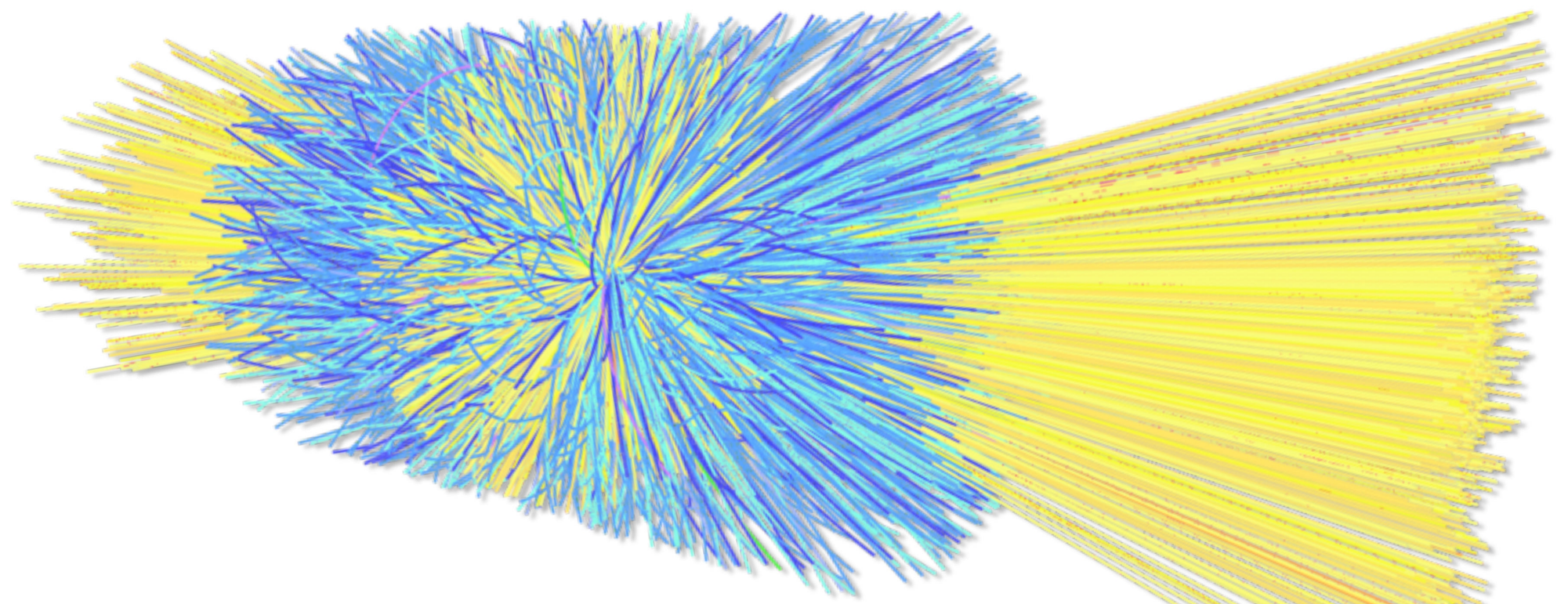

- At the HL-LHC (upgrade phase 2) we expect $\mu=200$ within a similar or shorter space.

- Higher track density requires a better Inner Tracker to maintain tracking and b-tagging performances.

- Existing components will be heavily radiation-damaged by LS3. 
- New Inner Detector will be called the 'Inner Tracker' (ITk).

- All sensors in the ITk will be Silicon-based.

$($ Red $=$ Silicon Strips, Blue $=$ Silicon Pixels $)$
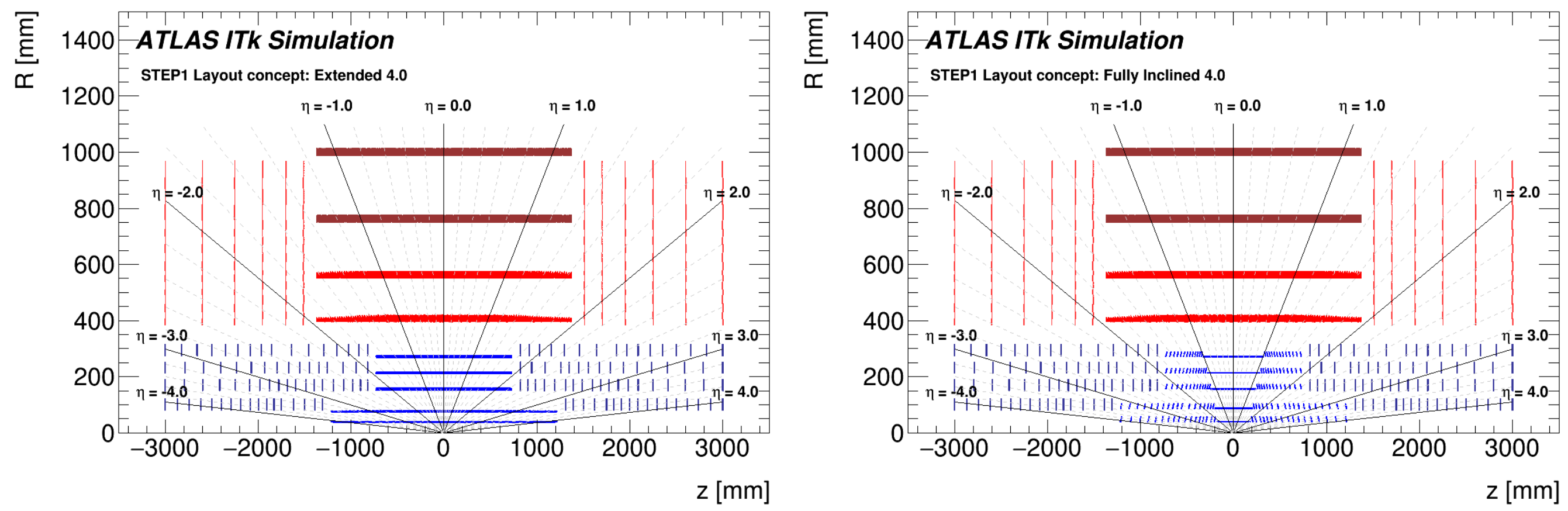

- We will also increase $\eta$ coverage to 3.2 or 4.0 (currently 2.5 in Run-2) to increase acceptance and reconstruct forward jets for VBF and VBS.

- ITk layout and design are not yet finalised: Four possible pixel barrel layouts are being considered. 
- Outside a radius of $362 \mathrm{~mm}$, the ITk will contain a Silicon strip detector: 4 barrel layers, 12 end-cap disks (6 per end), each disk built up of 9 'petals'.

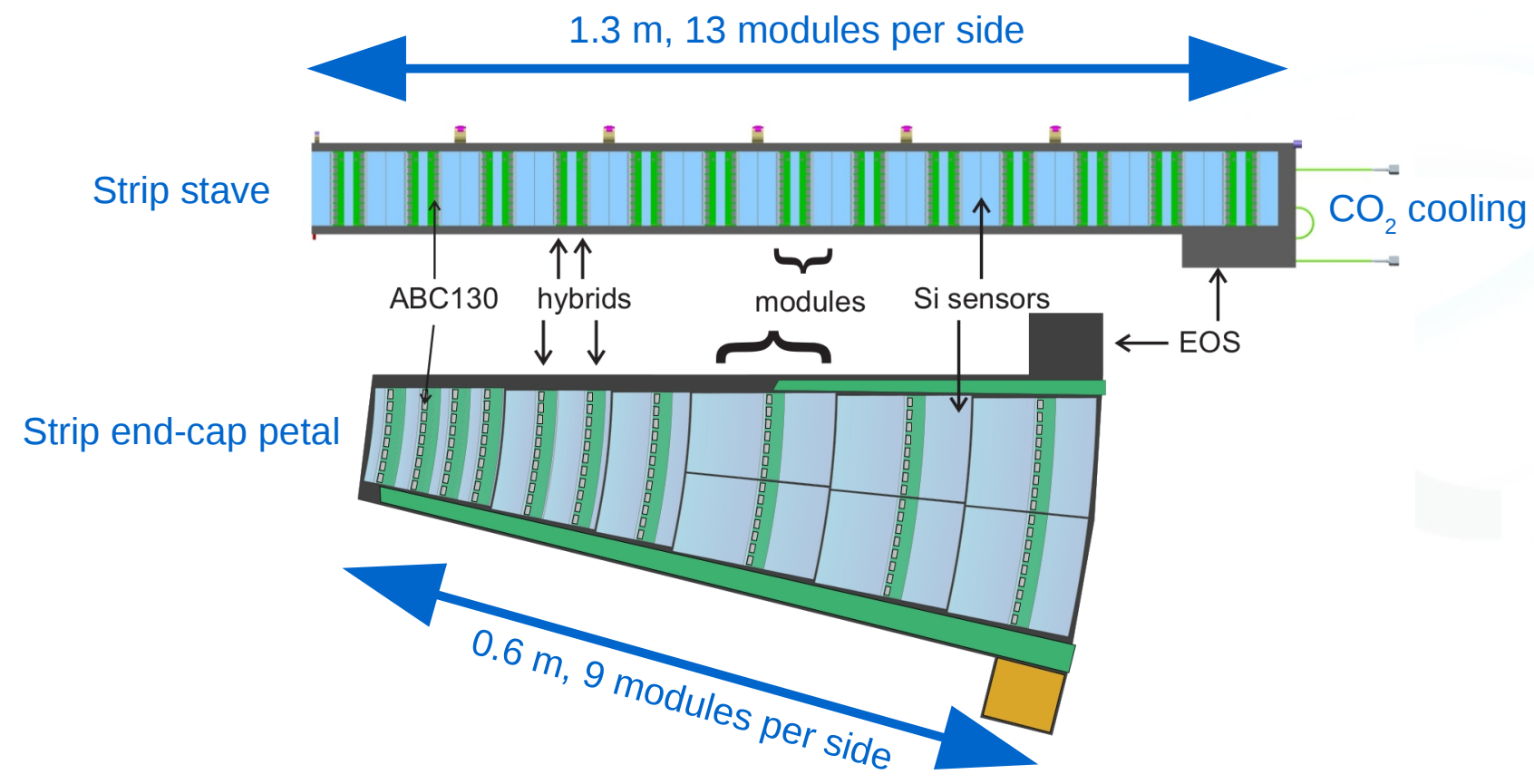

- Staves and end-cap petals have modules on both sides to allow overlap.

- Modular design eases large-scale production.

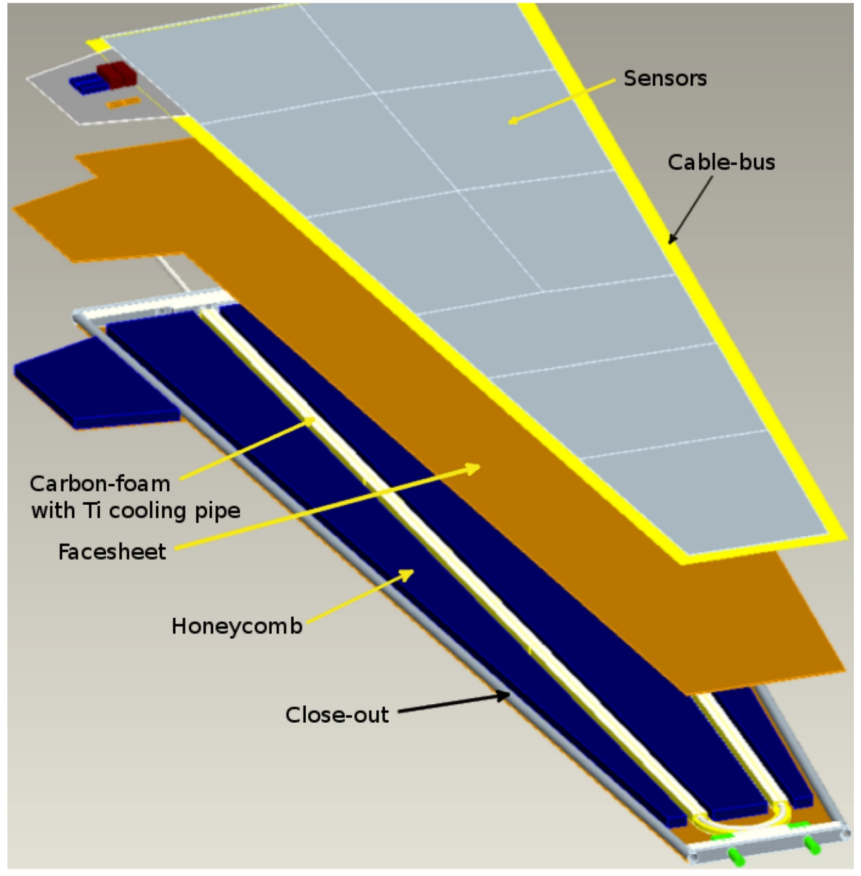

(Strips end-cap petal components) 
- Pixel end-caps will consist of rings in four layers, (at different radii).

- Each ring consists of a carbon core containing cooling and electrical services. Each ring is built out of two half-rings, for ease of construction.

- Modules are mounted on both sides of each ring to allow overlap.

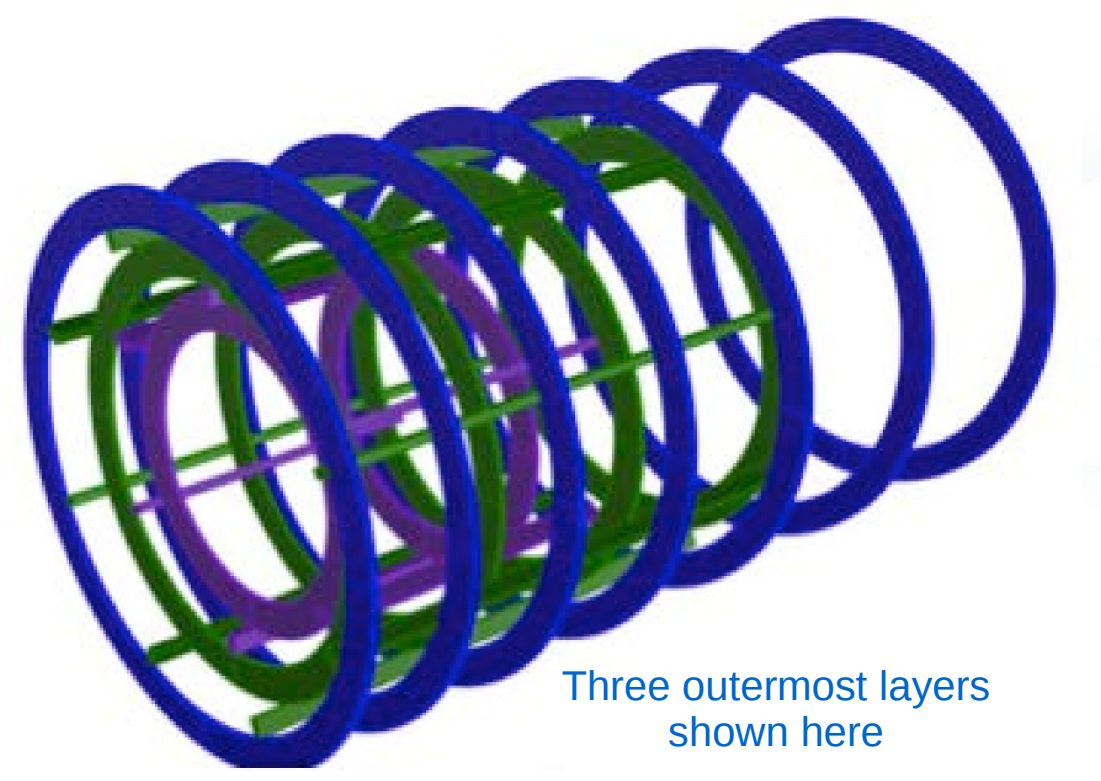

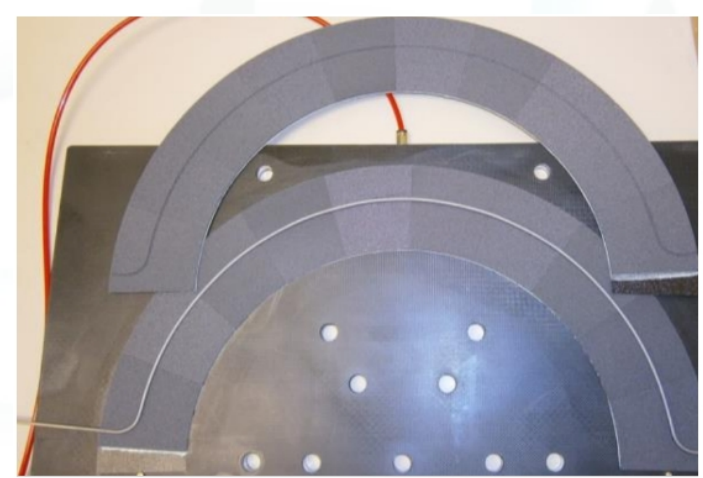

Titanium CO2 cooling pipe embedded in ring.

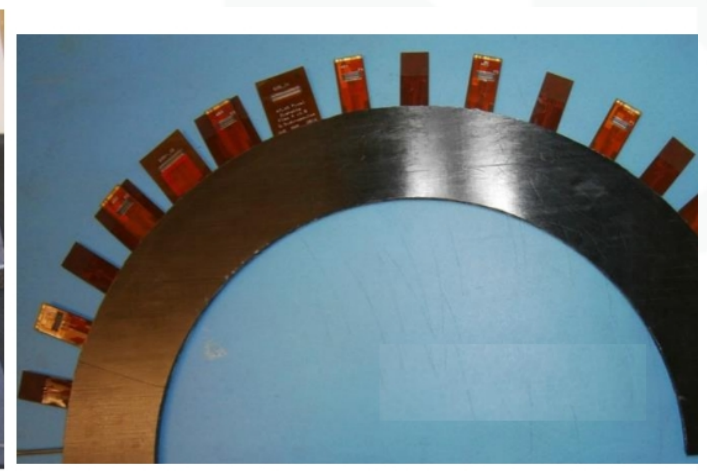

Electrical services (flex) embedded in ring.

- Innermost pixel end-cap ring layer needs to be more robust due to higher radiation dosage.

- Number of rings per layer is still under optimisation. 


\section{Inner Detector Upgrades: Pixel Barrel}

- Four layouts are under consideration for the ITk pixel barrel: Two 'extended' layouts (sensors parallel or perpendicular to beam line) Two 'inclined' layouts (with sensors also angled towards the beam-spot)

Extended

$\mid$ Eta $\mid<3.2$
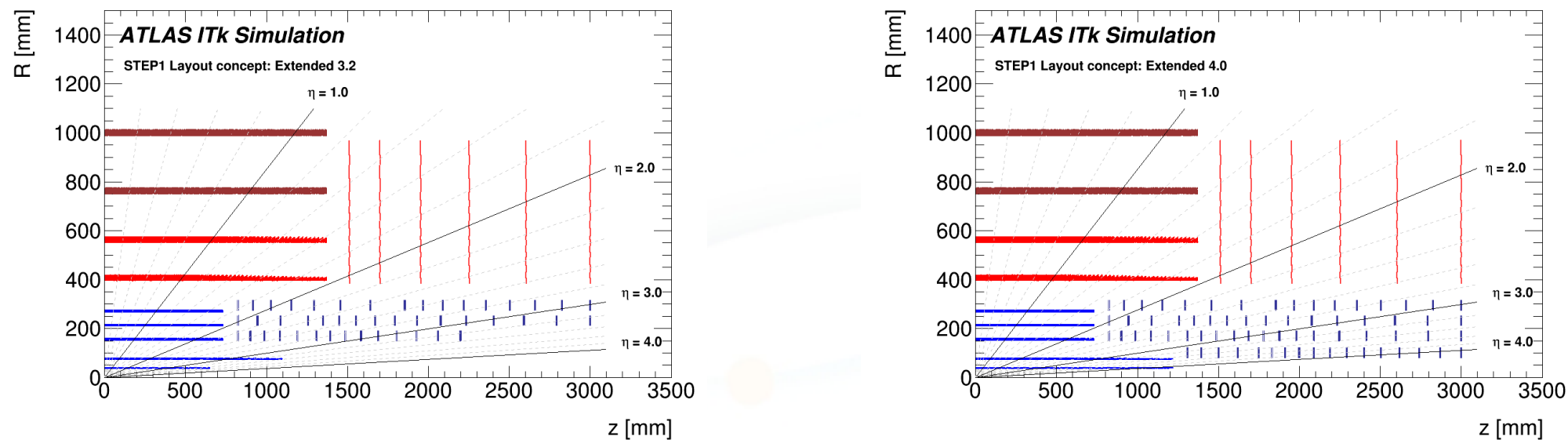

Extended

$\mid$ Eta $\mid<4$
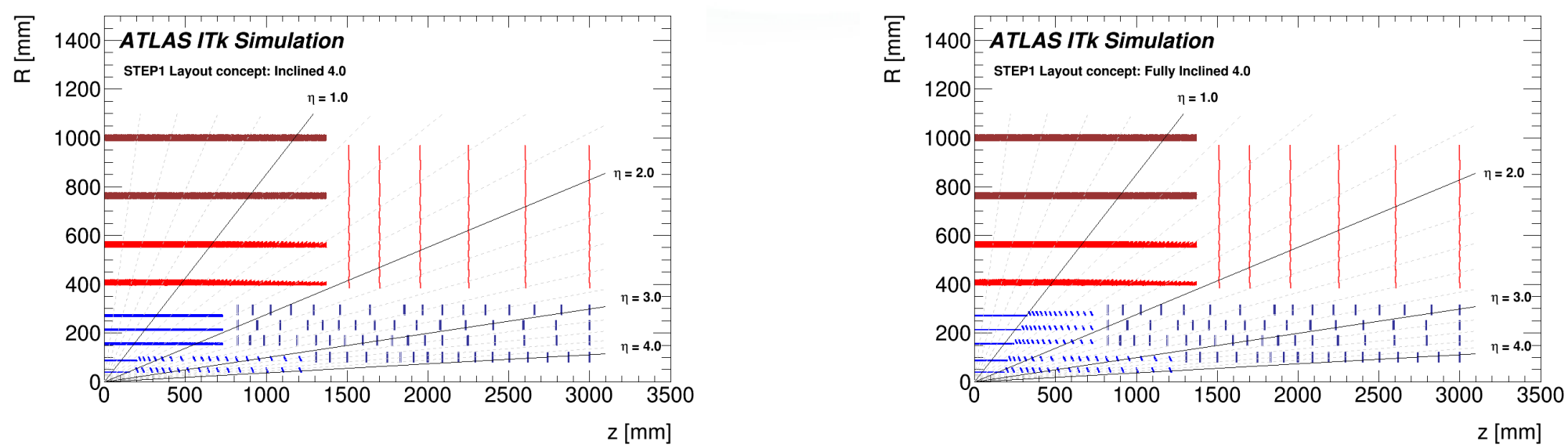

Fully Inclined

$\mid$ Eta $\mid<4$

$\mid$ Eta $\mid<4$

- All layouts consist of five pixel barrel layers 
- Four layouts are under consideration for the ITk pixel barrel: Two 'extended' layouts (sensors parallel or perpendicular to beam line) Two 'inclined' layouts (with sensors also angled towards the beam-spot)

Extended $\mid$ Eta $\mid<3.2$
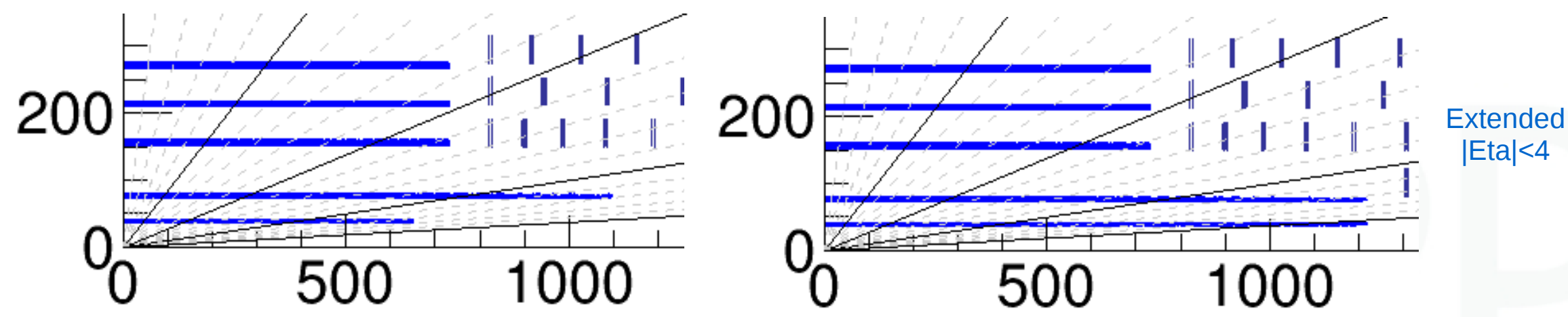
$\underset{\mid E \text { ta } \mid<4}{\text { Partially Inclined }} 200$
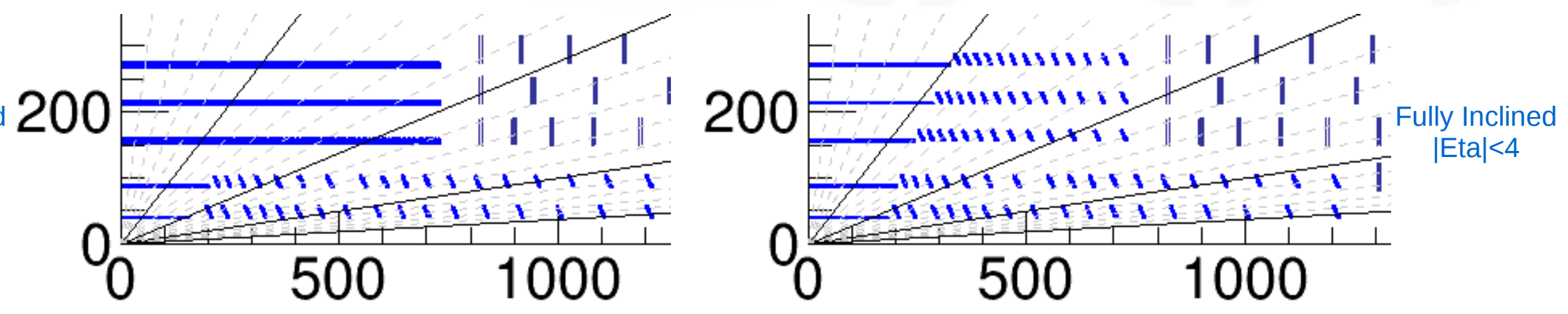

- All layouts consist of five pixel barrel layers 
- Inclined layouts aim to have sensors at more optimum angles to (more perpendicular to) incoming particles, thus reducing material and cluster size to improve track resolutions and identification, (and cost).

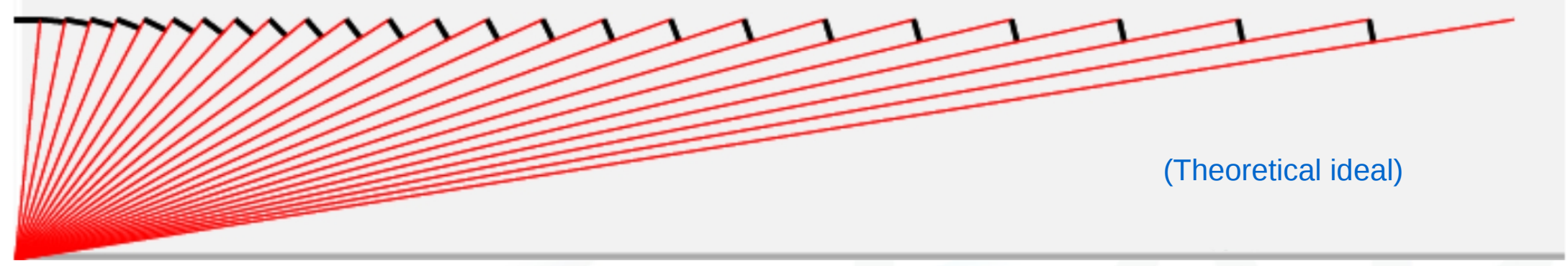

Non-inclined layouts have single transition region between parallel-to-beamline and perpendicular-to-beamline sensor regions.
Inclined layouts aim to improve on this by inclining sensors towards interaction point in this transition region.

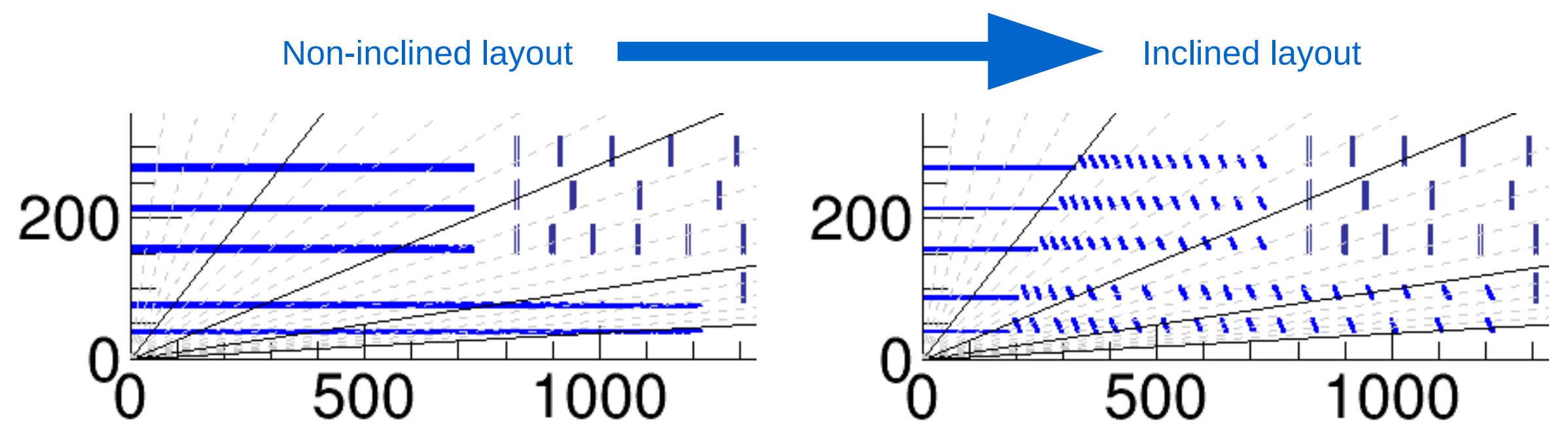


- Inclined layouts have multiple options for inclined sensor support.

- Layers to be joined in pairs: each stave would support two layers of pixel sensors, leading to increased stiffness.
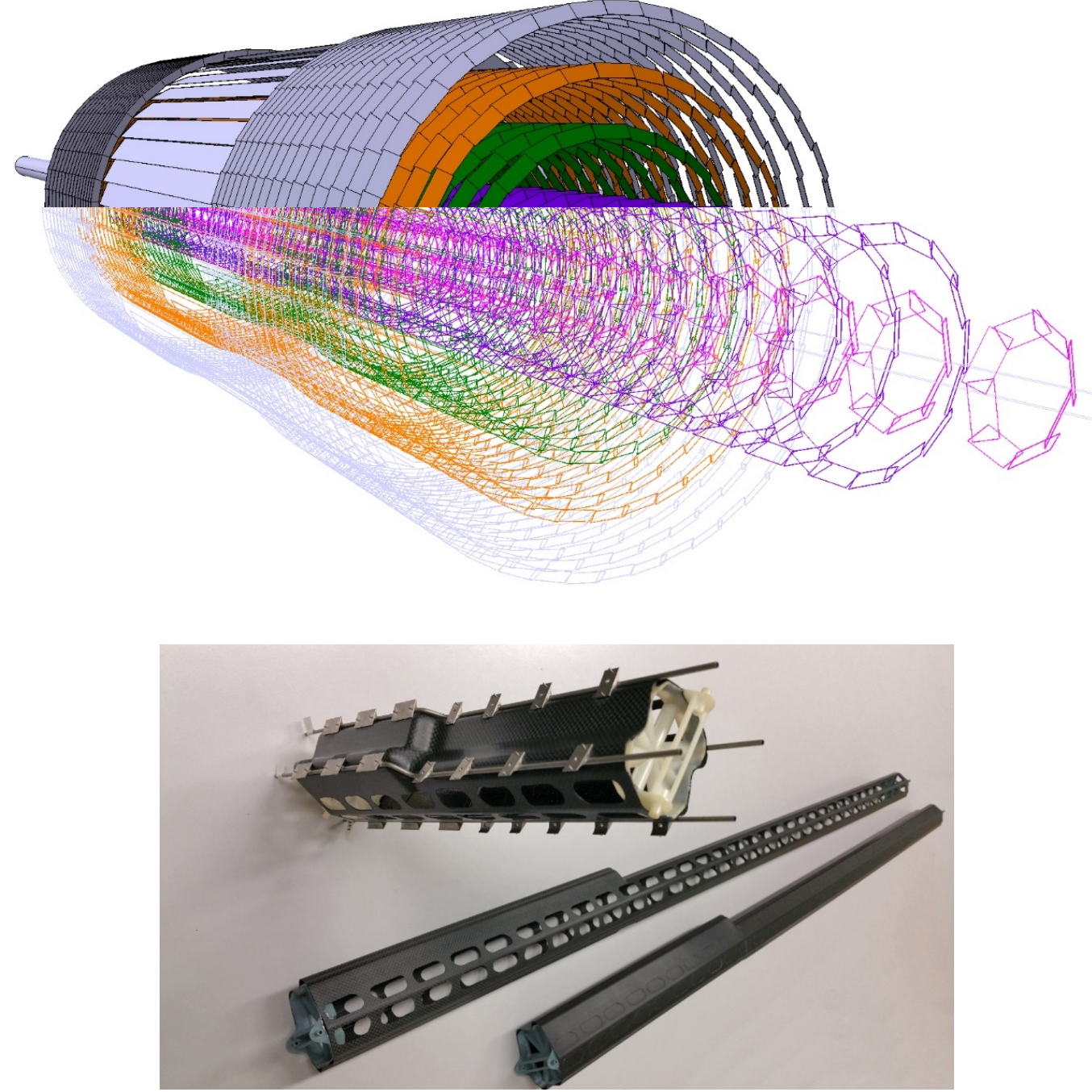

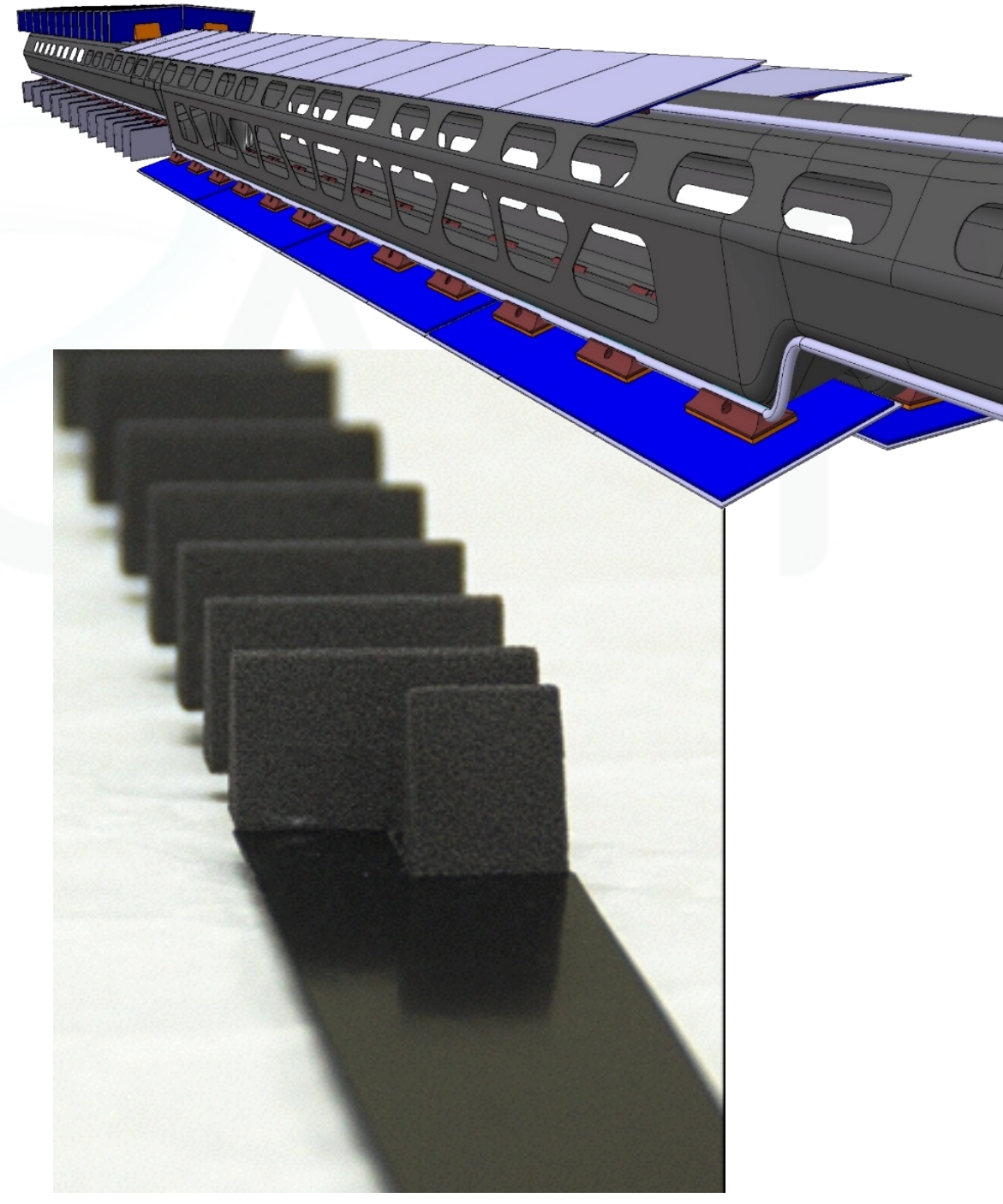


- With extended layouts, high-n particles will pass through pixel sensors at shallow angles, creating elongated clusters.
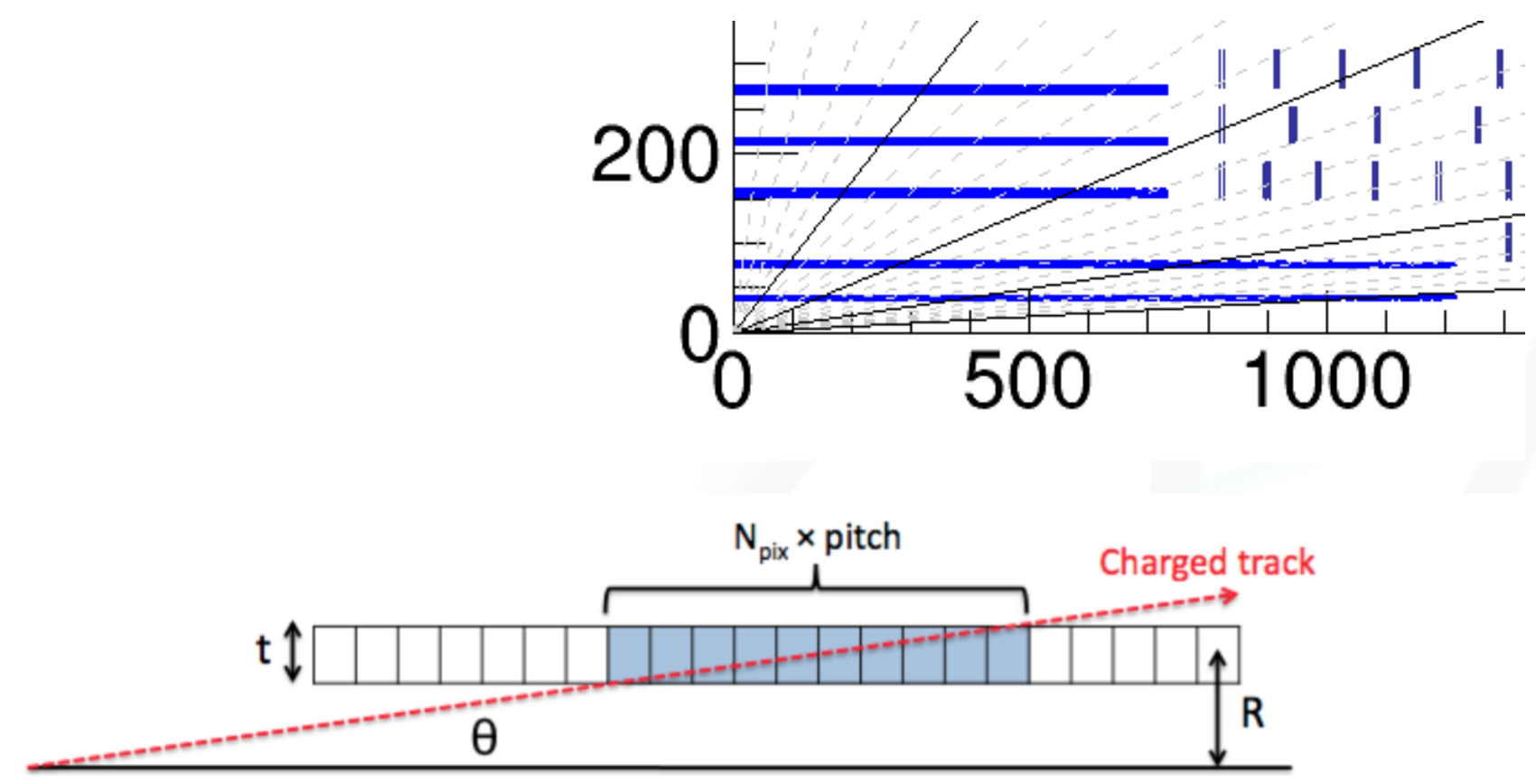

$$
N_{p i x}=\frac{t}{\tan \theta \times p}
$$

LHC beam line

- The aim is to then use cluster length to determine incident angle of incident particle.

- In this way, a single cluster becomes a 4D spacepoint.

- This information can then be fed into track fitting to improve track resolutions and identification. 
- Extended layouts also involve joining pairs of pixel layers with support structure, for increased stiffness:

Each stave would hold modules for two layers.

- Also looking at carbon pipes for $\mathrm{CO}_{2}$ cooling pipes, to reduce mass and increase radiation length of the staves. (Default $\mathrm{CO}_{2}$ cooling tube material is Titanium.)
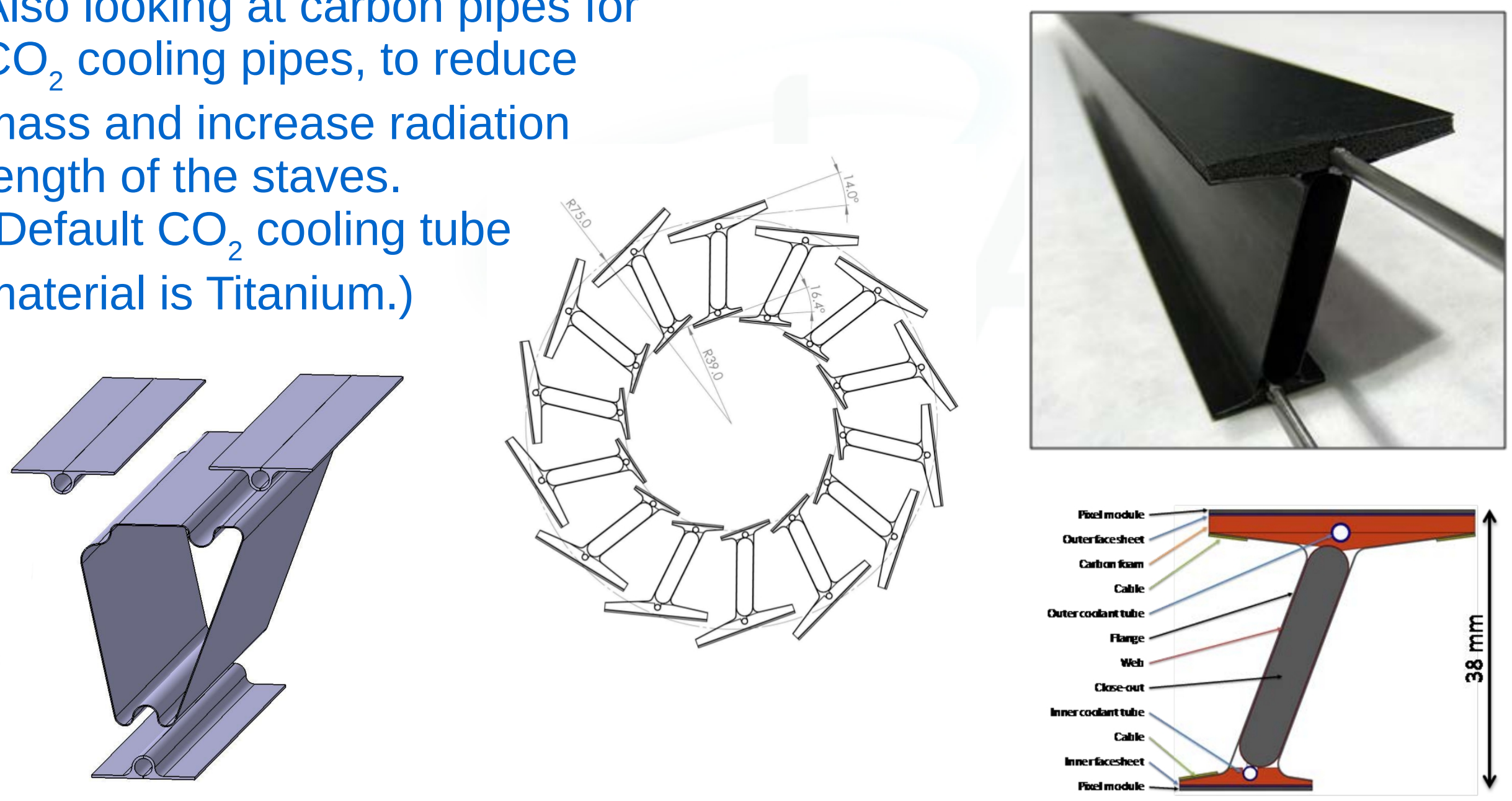
- ITk has a significant reduction in material (and so $X / X_{0}$ ) compared to current Run-2 Inner Detector.

- Largest source of $X / X_{0}$ in current ITk layouts comes from services and cooling pipes for pixel end-caps, (not in the pixel end-cap rings themselves, but in the services and cooling pipes connecting the end-cap layers to the outer boundaries of the ITk volume).

- ITk pixel end-caps still under optimisation to improve upon this $\mathrm{X} / \mathrm{X}_{0}$ issue.

For comparison,

$\mathrm{X} / \mathrm{X}_{0}$ of current Run-2 Inner Detector:

at $\eta=0, \quad X / X_{0}=0.5$

at $\eta=1.5, X / X_{0}=2.5$

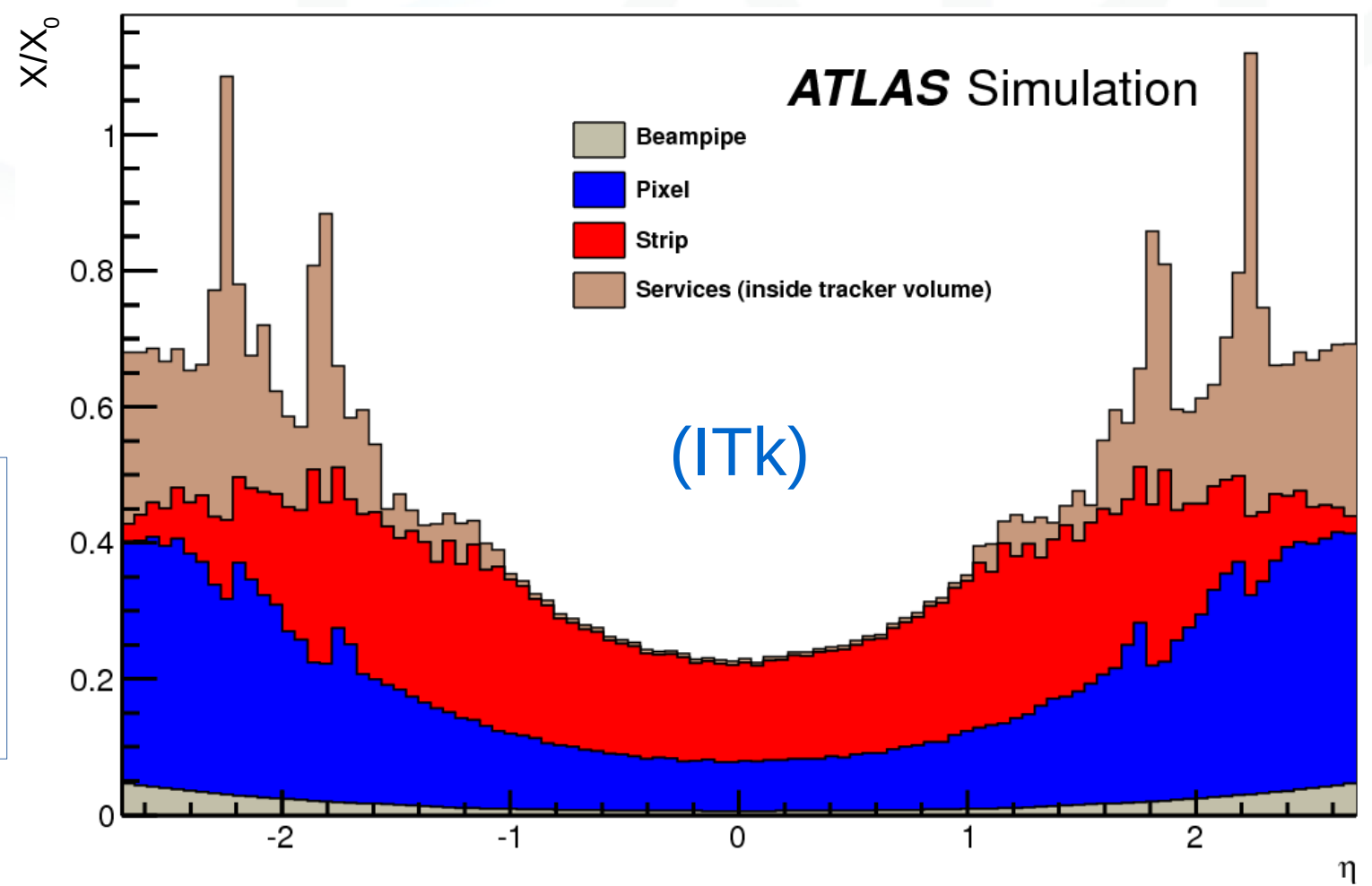


- Pixel sensors must be kept sufficiently cool to avoid thermal runaway.

- Particularly challenging for inclined designs.

- Lowering $\mathrm{CO}_{2}$ temperature becomes more costly at lower temperatures.

- Pixel staves and rings have been tested, with heaters in place of pixel sensors, to ensure that they allow for efficient cooling, with cooling efficiency quantified by a 'Thermal Figure Of Merit' (TFOM):

$$
\mathrm{TFOM}=\frac{\Delta T}{P_{\text {heater }}} A_{\text {heater }}
$$

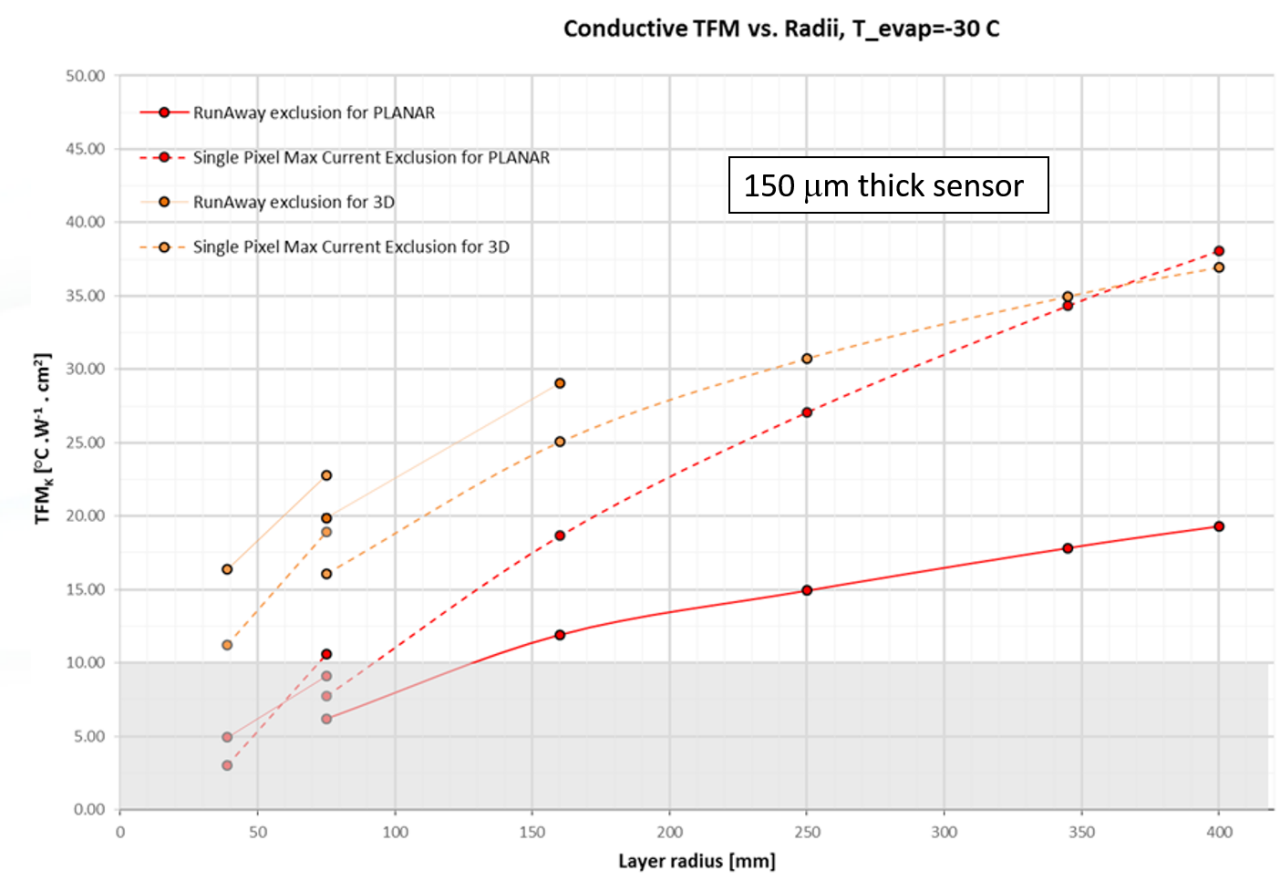

- Measured temperature difference in TFOM is not only due to heater power, (blackbody radiation also present, for example), so modified TFOM definition has been proposed to account for 'parasitic' heat sources:

$$
\mathrm{TFOM}=\frac{\Delta T}{P_{\text {heater }}+P_{\text {parasitic }}} A_{\text {heater }} \quad\left[{ }^{\circ} \mathrm{C} \mathrm{cm}^{2} / \mathrm{W}\right]
$$


- ITk pixel detector will require $5 \mathrm{gbps}$ data read-out rate.

- Can currently achieve 6.25Gbps.

- Choice of technology to use: Twin-axial or Flex.

- Twin-axial is simpler to produce.

- Flex contains less material (so has less X/X $\mathrm{X}_{0}$ ).
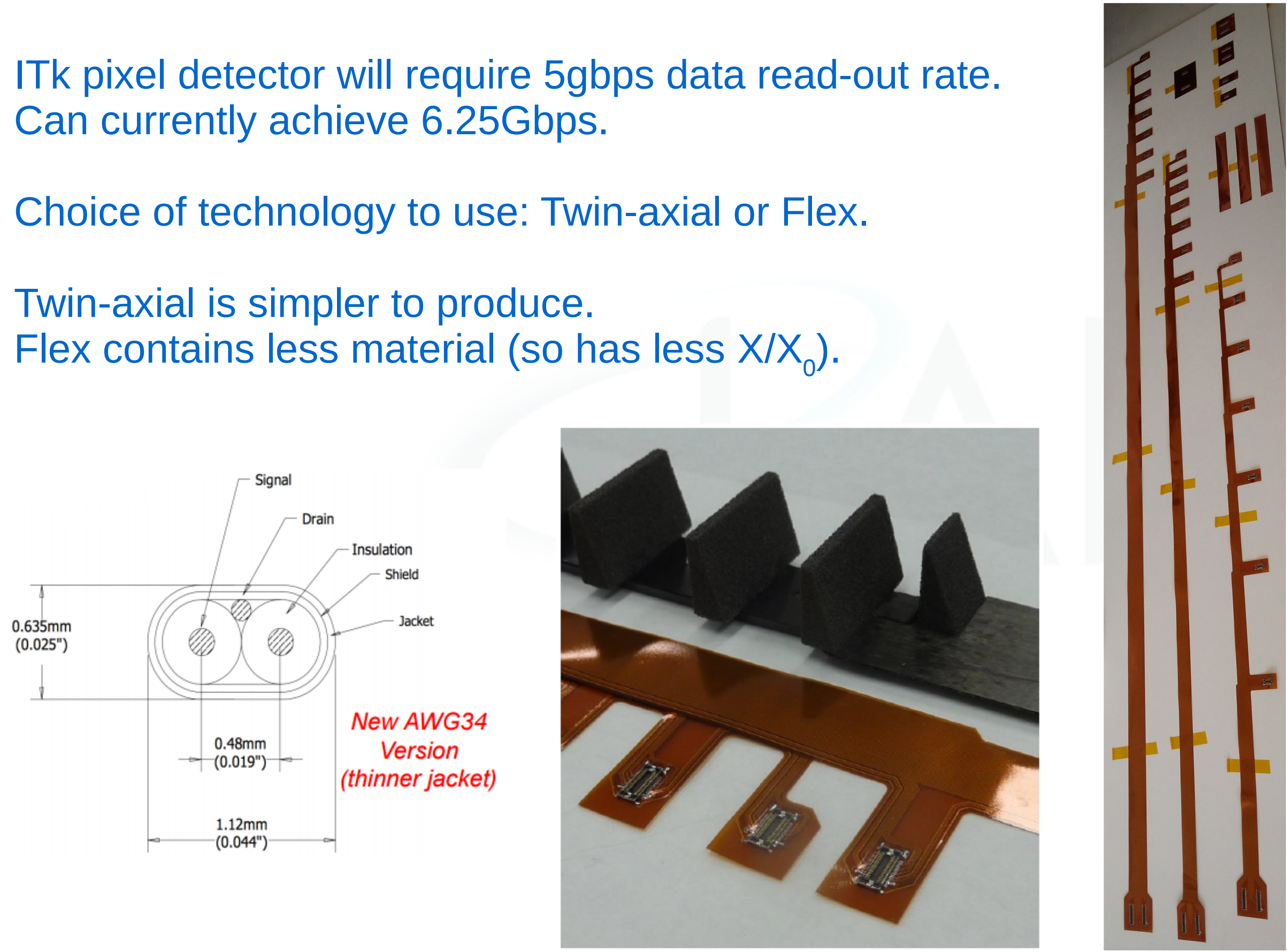
ATLAS Inner Tracker (ITk) will replace the current ATLAS Inner Detector for the HL-LHC

ITk will be an all-Silicon sensor design, with both Silicon pixel and Silicon strips, going to $|\eta|<4$ (or $|\eta|<3.2$ )

Reduction of material and improved tracking performance compared to current Run-2 ATLAS Inner Detector

Several layout designs being considered - all novel. Further layout optimisation and design qualification to be performed

Decision on which layout to use will be made early 2017. Pixel TDR due late 2017

ATLAS, with ITk, will start taking data from the HL-LHC in 2026 


\section{Backup}


- These upgrades will improve our measurements and searches:

Run 1

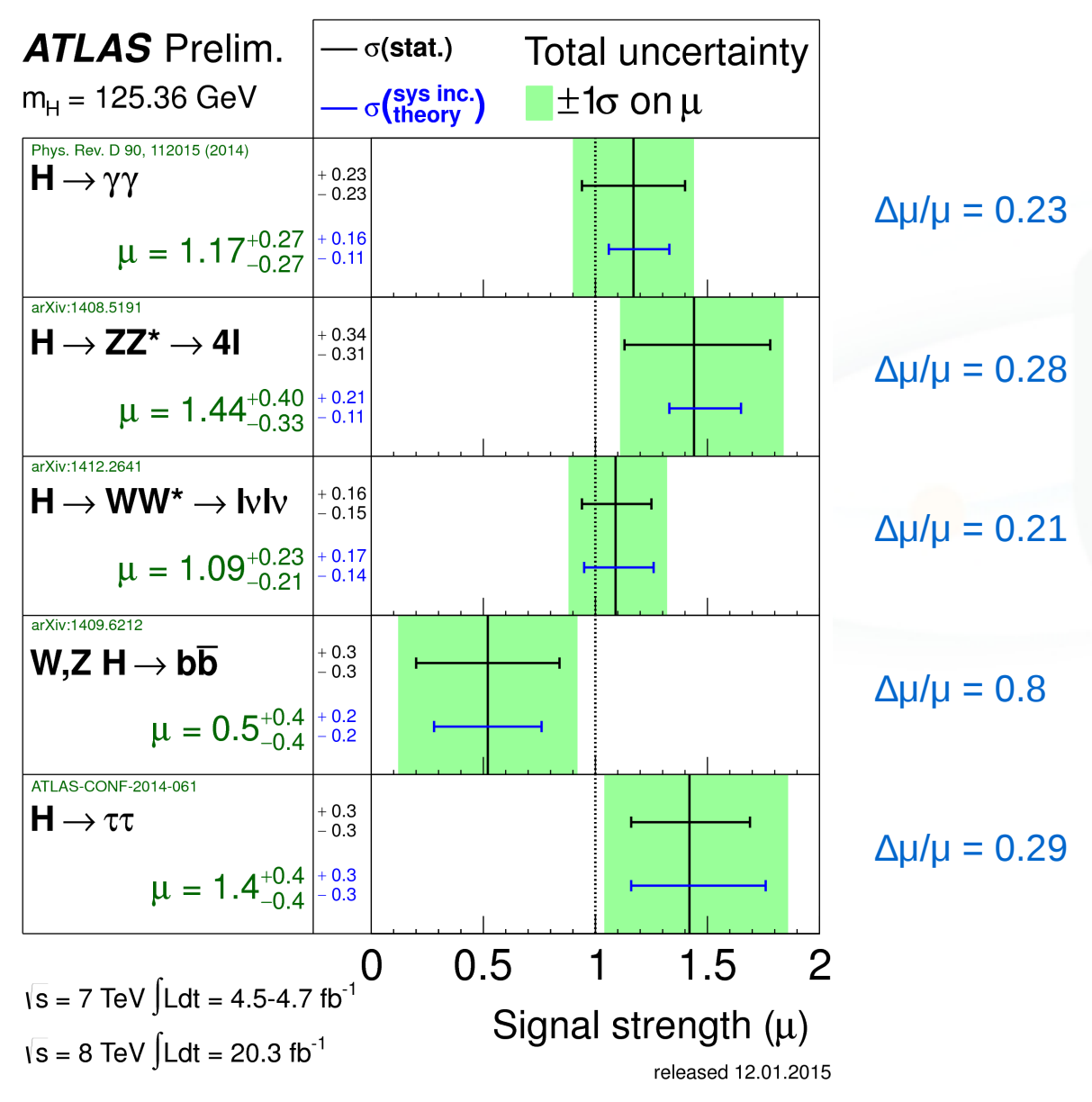

Run 3 and HL-LHC

ATLAS Simulation Preliminary

Is = $14 \mathrm{TeV}: \int \mathrm{Ldt}=300 \mathrm{fb}^{-1} ; \int \mathrm{Ldt}=3000 \mathrm{fb}^{-1}$

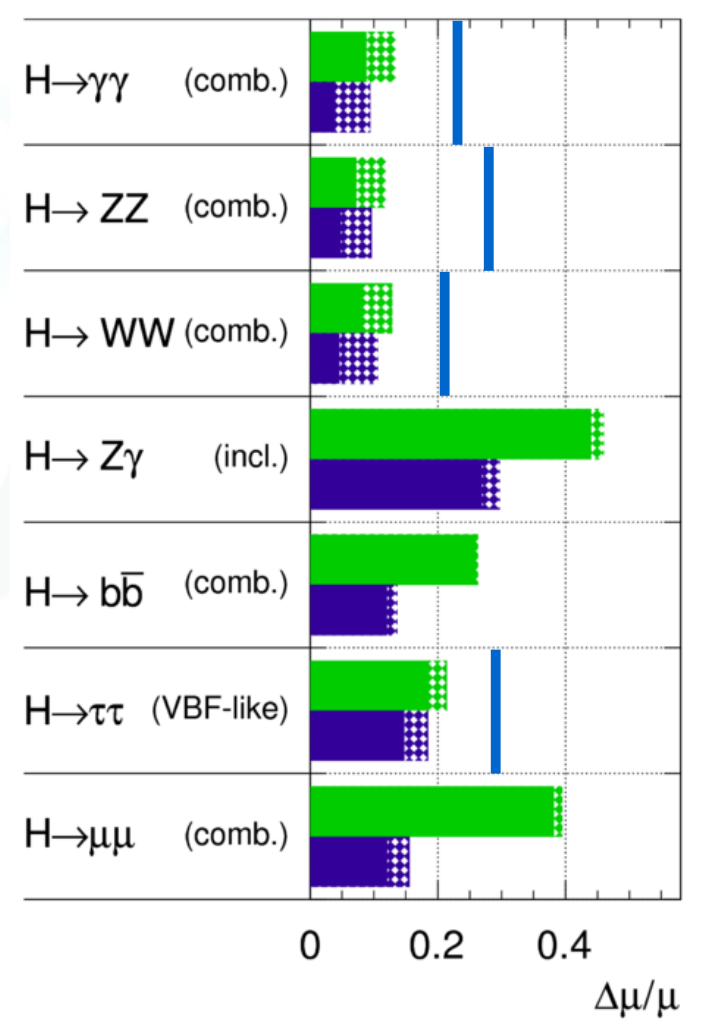

Dashed areas indicate $\Delta \mu / \mu$ with current theory uncertainties.

Vertical blue lines are Run 1 values. 

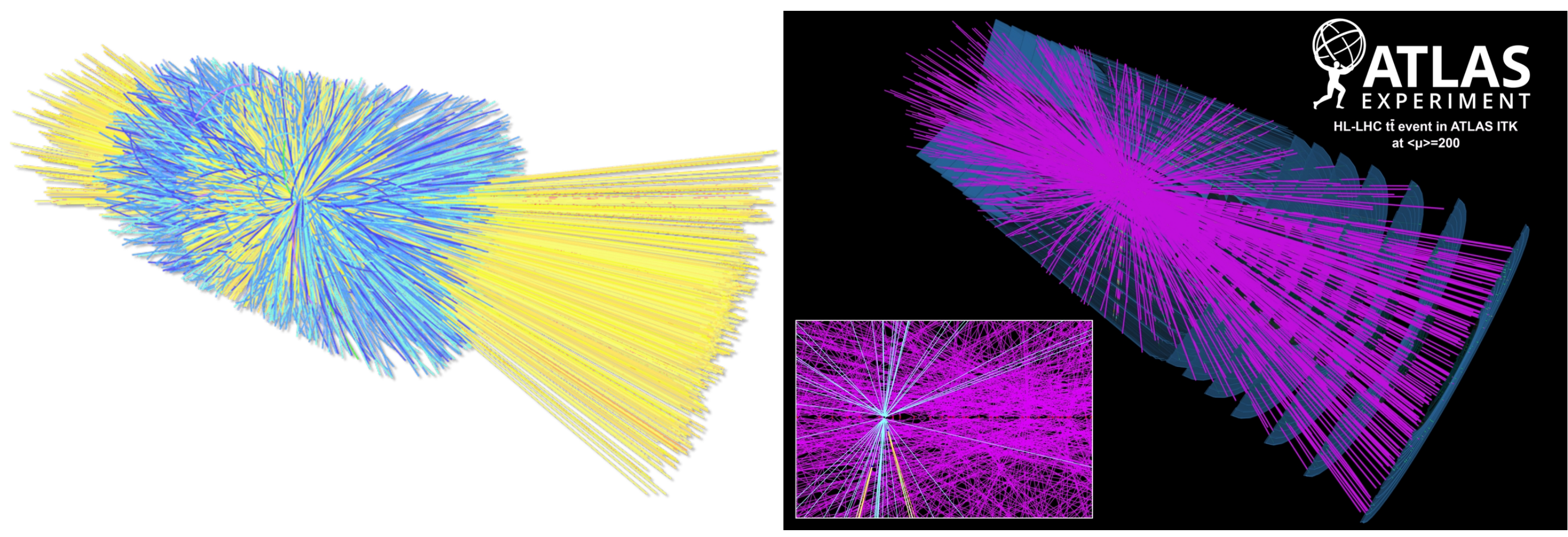
- Extended layouts also involve joining pairs of pixel layers with support structure, for increased stiffness: Each stave would hold modules for two layers.
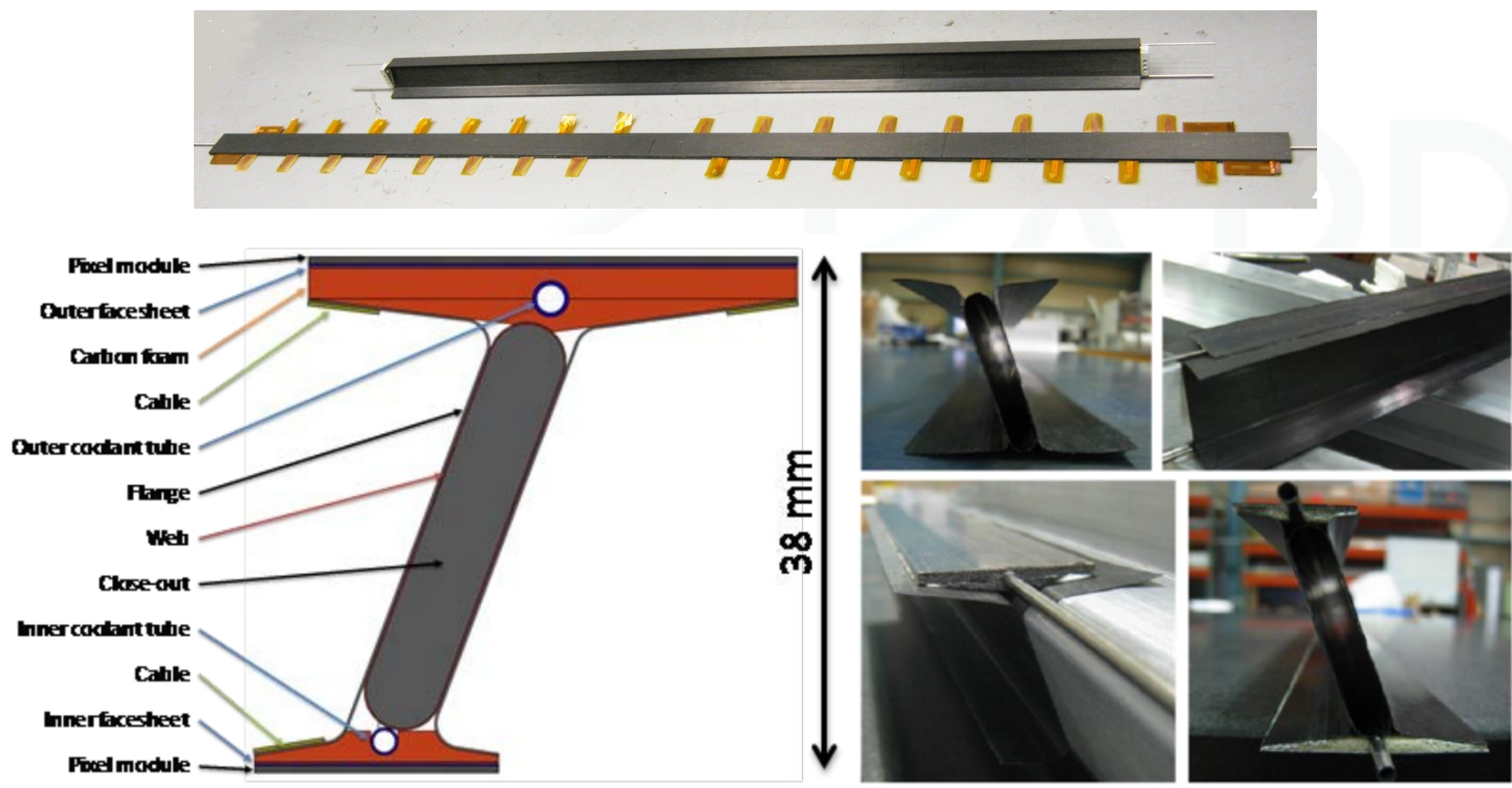


\section{Inner Detector Upgrades: Related Talks}

- Operational Experience with the ATLAS Pixel detector

- The ATLAS Insertable B-Layer: from construction to operation

- Physics performance of the ATLAS Pixel Detector

- ATLAS track reconstruction in dense environments and its inefficiency measurements using pixel DE/DX

- Primary vertex reconstruction with the ATLAS detector

- Toward HL-LHC: strategies for DAQ and track triggers

- 4D fast tracking for experiments at HL-LHC

- From LHC to HL-LHC...challenges and routes to solutions

- CMS Pixel Detector design for HL-LHC

- 3D sensors for the HL-LHC

- Optimization of thin n-in-p planar pixel modules for the ATLAS upgrade at HL-LHC

- Test beam results of 3D detectors constructed with single-crystal and poly-crystalline diamond

- Radiation damage caused by neutron capture in boron doped silicon pixel sensors

- Application of a Transient-Current-Technique based on a Two- Photon-Absorption process to the characterization of a HV-CMOS deep n-well

- Development of CdTe Pixel Detectors Combined with an Aluminum Schottky Diode Sensor and Photon-Counting ASICs

- Overview and perspectives of HR CMOS

- Status of HVCMOS Sensor Developments for ATLAS

- Total Ionization Dose effects in the FE-I4 front-end chip of the ATLAS Pixel IBL detector

- Radiation-Hard/High-Speed Parallel Optical Links

- Ultra-light mechanical supports for pixel detectors

- The Atlas IBL CO2 Cooling System

- 3D stacking technology

- Development of n+-in-p planar pixel sensor flip-chip modules with quad FE-I4 readout ASIC's

- Pixel hybridization technologies for the HL-LHC

- Thin hybrid pixel assembly with backside compensation layer on roic

- Poster sessions

$\bullet$

$\bullet$ 